\title{
ARTICLE
}

Received 29 Jan 2013 | Accepted 26 Mar 2013 | Published 30 Apr $2013 \quad$ DOl: 10.1038/ncomms2800

\section{Ultraviolet-B-mediated induction of protein-protein interactions in mammalian cells}

\author{
Remco P. Crefcoeur ${ }^{1}$, Ruohe Yin², Roman Ulm² \& Thanos D. Halazonetis ${ }^{1,3}$
}

Light-sensitive proteins are useful tools to control protein localization, activation and gene expression, but are currently limited to excitation with red or blue light. Here we report a novel optogenetic system based on the ultraviolet-B-dependent interaction of the Arabidopsis ultraviolet-B photoreceptor UVR8 with COP1 that can be performed in visible light background. We use this system to induce nuclear accumulation of cytoplasmic green fluorescent protein fused to UVR8 in cells expressing nuclear COP1, and to recruit a nucleoplasmic red fluorescent protein fused to COP1 to chromatin in cells expressing UVR8-H2B. We also show that ultraviolet-B-dependent interactions between DNA-binding and transcription activation domains result in a linear induction of gene expression. The UVR8-COP1 interactions in mammalian cells can be induced using subsecond pulses of ultraviolet-B light and last several hours. As UVR8 photoperception is based on intrinsic tryptophan residues, these interactions do not depend on the addition of an exogenous chromophore.

\footnotetext{
${ }^{1}$ Department of Molecular Biology, University of Geneva, 30 quai Ernest-Ansermet, CH 1205 Geneva, Switzerland. ${ }^{2}$ Department of Botany and Plant Biology, University of Geneva, 30 quai Ernest-Ansermet, CH 1205 Geneva, Switzerland. ${ }^{3}$ Department of Biochemistry, University of Geneva, 30 quai Ernest-

Ansermet, CH 1205 Geneva, Switzerland. Correspondence and requests for materials should be addressed to T.D.H. (email: thanos.halazonetis@unige.ch).
} 
$\mathrm{n}$ optogenetics, proteins are engineered to contain a photoreceptor that triggers functionality when struck by a photon of a specific wavelength. Optogenetics has been mainly applied in the neurosciences, where stimulation of microbial opsins by light polarizes the membrane, offering temporally precise activation of defined neurons ${ }^{1}$. In the past years, the interest in optogenetics has expanded to other fields, with both red and blue lightsensitive proteins having been engineered to control protein localization, pathway activation and gene expression ${ }^{2-6}$. Yet, for versatility in experimental design, there is a need for development of multiple optogenetic systems, especially systems that do not require addition of exogenous chromophores and that respond to different wavelengths of light.

In Arabidopsis thaliana, ultraviolet-B (280-315 nm) light induces an interaction of the photoreceptor UVR8 with its partner COP1 (refs 7,8). Biochemical and structural data indicate that UVR8 contains a seven bladed $\beta$-propeller type domain that in the absence of ultraviolet-B forms a homodimer ${ }^{7,9,10}$. The dimer interface is rich in tryptophan (Trp) residues, several of which are physically close to each other. When excited by ultraviolet-B, the Trp aromatic residues, particularly Trp285, function as a chromophore triggering monomerization of UVR8 (refs 7,9,10). Mutant versions of UVR8 with Trp285 to phenylalanine $\left(\mathrm{UVR} 8^{\mathrm{W} 285 \mathrm{~F}}\right)$ or alanine $\left(\mathrm{UVR} 8^{\mathrm{W} 285 \mathrm{~A}}\right)$ substitutions do not respond to ultraviolet-B and are in vivo constitutively dimeric and monomeric, respectively ${ }^{7}$. Upon monomerization, UVR8 interacts with the E3 ubiquitin ligase COP1 and the two proteins accumulate in the nucleus, where they regulate expression of genes that provide protection from ultraviolet-B (refs 8,11,12). COP1 contains three domains that are important for its function: an N-terminal RING domain, a coiled-coil domain and a seven WD-40 repeat domain at its C-terminus ${ }^{13}$. The C-terminal 340 amino acids of COP1 $\left(\mathrm{COP} 1^{\mathrm{C} 340}\right)$, corresponding to the WD-40 domain, are sufficient to interact with UVR8 in yeast ${ }^{7}$.

Here, we describe a novel optogenetic system that utilizes the UVR8 and COP1 interacting modules to induce ultraviolet-Bdependent protein-protein interactions. This new system does not require an exogenous chromophore and utilizes a wavelength that does not interfere with the commonly used fluorescent reporter proteins.

\section{Results}

Ultraviolet-B induced protein translocation. To setup a novel optogenetic system, we first examined whether UVR8 and COP1 ${ }^{\mathrm{C} 340}$ interact in a ultraviolet-B-dependent manner in mammalian cells. We fused UVR8 to green fluorescent protein a

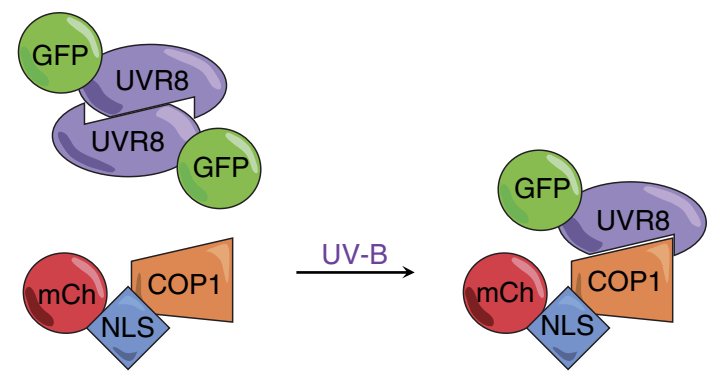

b

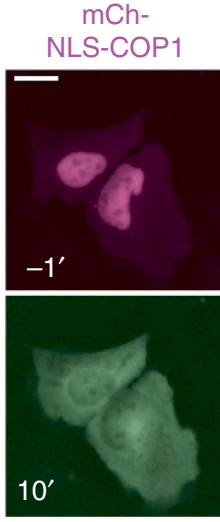

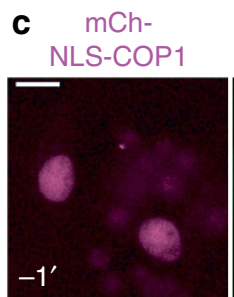

$\mathrm{mCh}-$

NLS-COP1

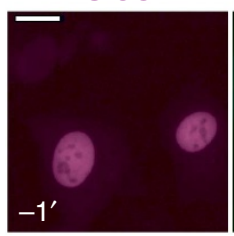

GFP-

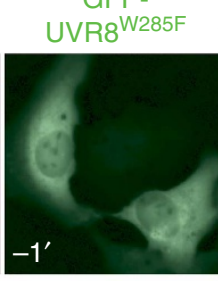

GFP.

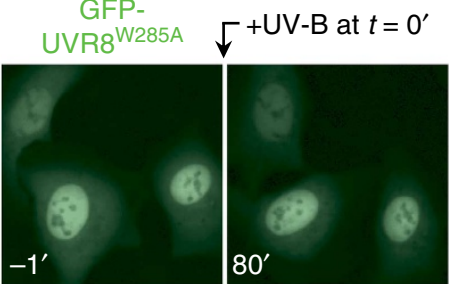

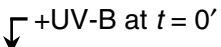

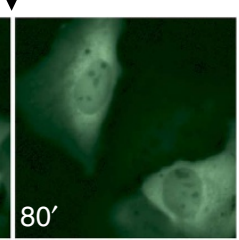

d

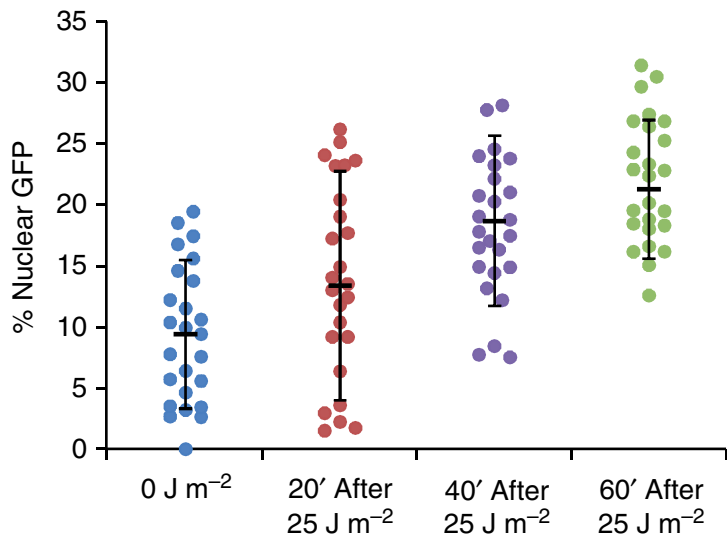

Figure 1 | Ultraviolet-B-dependent nuclear retention of GFP-UVR8. (a) Schematic representation of the ultraviolet-B-induced interaction of GFP-UVR8 with $\mathrm{mCh}-\mathrm{NLS}-\mathrm{COP}{ }^{\mathrm{C} 340}$. As a homodimer, GFP-UVR8 does not interact with $\mathrm{mCh}-\mathrm{NLS}-\mathrm{COP} 1^{\mathrm{C} 340}$. After exposure to ultraviolet-B, the GFP-UVR8 dimer dissociates into monomers, each of which can interact with mCh-NLS-COP1 C340. (b) Localization of GFP-UVR8 and mCh-NLS-COP1C340 in U2OS cells before and after exposure to ultraviolet-B $\left(25 \mathrm{Jm}^{-2}\right)$. Timepoints in minutes, relative to the ultraviolet-B pulse, are indicated. Scale bar, $20 \mu \mathrm{m}$. (c) U2OS cells expressing GFP-UVR8 ${ }^{\mathrm{W} 285 \mathrm{~F}}$ or GFP-UVR $8^{\mathrm{W} 285 \mathrm{~A}}$ and $\mathrm{mCh}-\mathrm{NLS}-\mathrm{COP} 1^{\mathrm{C} 340}$ before and after a ultraviolet-B pulse (25 J m ${ }^{-2}$ ). Timepoints in minutes, relative to the ultraviolet-B pulse are indicated. Scale bar, $20 \mu \mathrm{m}$. (d) Fraction of nuclear GFP-UVR8 determined from analysis of live cell images before and after exposure to ultraviolet-B light. For each condition, 25 cells were analysed; circles indicate the values for each cell and lines show the means \pm 1 s.d. of the mean. 
a
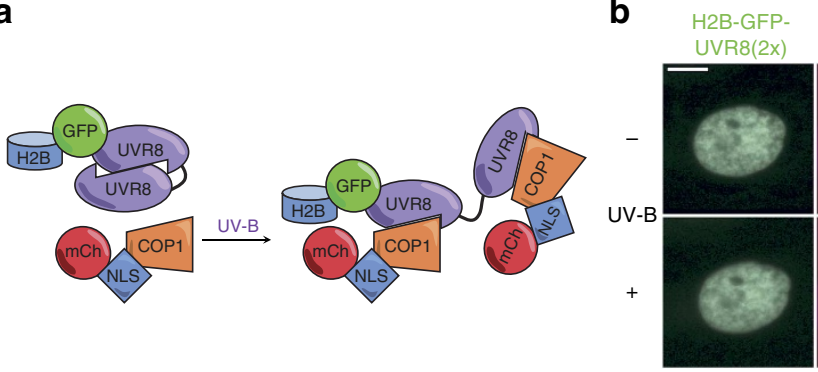
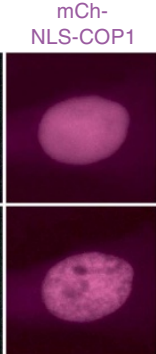

Merge

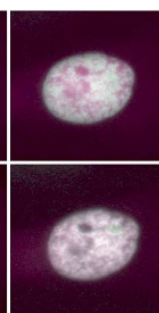

C

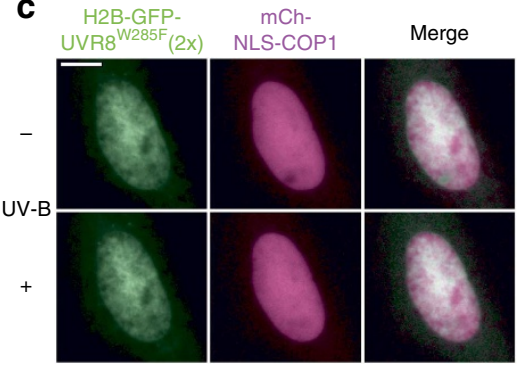

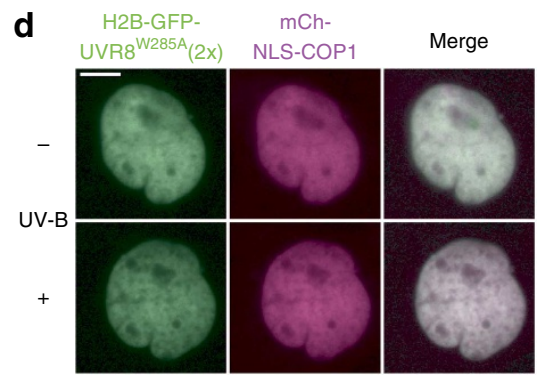

e

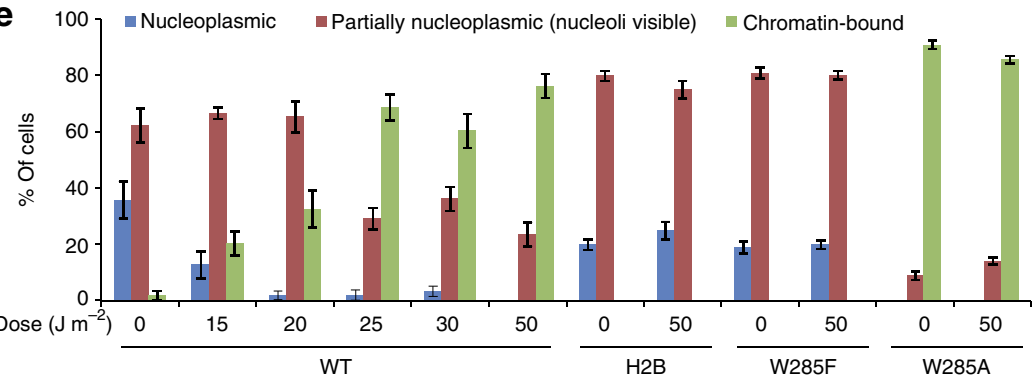

Figure 2 | Ultraviolet-B-dependent recruitment of $\mathbf{m C h}-\mathbf{N L S}-\mathbf{C O P 1} \mathbf{C 3 4 0}^{\mathbf{C}}$ to chromatin. (a) Schematic representation of the ultraviolet-B-induced interaction of H2B-GFP-UVR8(2x) with mCh-NLS-COP1 ${ }^{\mathrm{C} 340}$. In the absence of ultraviolet-B, the two UVR8 domains of H2B-GFP-UVR8(2x) interact with each other. After exposure to ultraviolet-B, this interaction is disrupted, allowing interaction with two mCh-NLS-COP1 ${ }^{\mathrm{C} 340}$ molecules. (b) Localization of H2B-GFP-UVR8(2x) and mCh-NLS-COP1 ${ }^{\mathrm{C} 340}$ before $(-)$ and after $(+)$ exposure to ultraviolet-B in U2OS cells. In this panel and in panels $\mathbf{c}, \mathbf{d}$ scale bar, $10 \mu \mathrm{m}$. (c) U2OS cells expressing mCh-NLS-COP1 ${ }^{\mathrm{C} 340}$ and H2B-GFP-UVR8 ${ }^{\mathrm{W} 285 \mathrm{~F}}(2 \mathrm{x})$ before $(-)$ and after $(+)$ ultraviolet-B illumination. (d) U2OS cells expressing $\mathrm{mCh}-\mathrm{NLS}-\mathrm{COP}_{1}{ }^{\mathrm{C} 340}$ and H2B-GFP-UVR8W285A $(2 \mathrm{x})$ before $(-)$ and after $(+)$ ultraviolet-B illumination. (e) Quantification of localization patterns of $m C h-N L S-C O P 1 C 340$ in U2OS cells co-expressing wild-type H2B-GFP-UVR8(2x) (WT), H2B-GFP (H2B) or the indicated H2B-GFP-UVR8(2x) mutants (W285A and W285F) before and after exposure to the indicated dose of ultraviolet-B. The bars indicate the means \pm 1 s.e. obtained from five independent experiments.

(GFP) and $\mathrm{COP} 1^{\mathrm{C} 340}$ to a monomeric Cherry (mCh) fluorescent protein bearing also a nuclear localization signal (NLS) (Fig. 1a, Supplementary Fig. S1). We then monitored the localization of GFP-UVR8 and mCh-NLS-COP1 $1^{\mathrm{C} 340}$ in U2OS cells by timelapse microscopy. Before ultraviolet-B illumination, GFP-UVR8 localized mostly in the cytoplasm, whereas mCh-NLS-COP1 ${ }^{\mathrm{C} 340}$ was nuclear. After illumination with ultraviolet-B, GFP-UVR8 gradually concentrated in the nucleus over the course of an hour (Fig. 1b). In contrast, under the same conditions, ultraviolet-B light did not induce nuclear retention of the GFP-UVR 8 W285F mutant, whereas GFP-UVR8 ${ }^{\mathrm{W} 285 \mathrm{~A}}$ was constitutively nuclear due to constitutive interaction with $\mathrm{mCh}-\mathrm{NLS}-\mathrm{COP} 1^{\mathrm{C} 340}$ (Fig. 1c). In cells that did not express mCh-NLS-COP ${ }^{\mathrm{C} 340}$, ultraviolet-B did not induce nuclear localization of GFP-UVR8 and GFP-UVR8 ${ }^{\text {W285A }}$ was constitutively cytoplasmic (Supplementary Fig. S2).

To quantitate the ultraviolet-B-induced nuclear accumulation of GFP-UVR8 in cells expressing mCh-NLS-COP1 ${ }^{\mathrm{C} 340}$, images of live cells were acquired at various times after exposure to a pulse of ultraviolet-B light and used to calculate the nuclear fraction of GFP-UVR8. The fraction of nuclear GFP-UVR8 increased over a 60-min time period after illumination with ultraviolet-B (Fig. 1d). As ultraviolet-B light induces an almost immediate UVR8 monomerization followed by interaction with COP1 (ref. 7), we speculate that the slow kinetics reflects inefficient transport of GFP-UVR8 from the cytoplasm to the nucleus, where it can be retained by $\mathrm{mCh}-\mathrm{NLS}-\mathrm{COP} 1^{\mathrm{C} 340}$.

As a second system to monitor ultraviolet-B-induced protein-protein interactions, we fused UVR8 to histone $\mathrm{H} 2 \mathrm{~B}$ and examined whether ultraviolet- $\mathrm{B}$ could be used to target nucleoplasmic COP $1^{\mathrm{C} 340}$-fusion proteins to chromatin. Our initial UVR8 construct contained a single UVR8 moiety fused to H2B-GFP (Supplementary Fig. S1). However, when we coexpressed H2B-GFP-UVR8 and $\mathrm{mCh}-\mathrm{NLS}-\mathrm{COP} 1^{\mathrm{C} 340}$ we observed constitutive interaction of the two proteins, independently of ultraviolet-B. We also observed constitutive interactions when mCh-NLS-COPC340 was coexpressed with H2B-GFP-UVR8 ${ }^{\mathrm{W} 285 \mathrm{~A}}$ and, even, with H2B-GFP-UVR8 ${ }^{\mathrm{W} 285 \mathrm{~F}}$ (Supplementary Fig. S3). We speculated that the internucleosome distance was too large to support dimerization of H2B-GFP-UVR8 molecules in the absence of ultraviolet-B. Thus, to allow the formation of UVR8 dimers, we fused H2B-GFP to two UVR8 moieties that were joined together by a 22-amino acid long flexible linker (Fig. 2a, Supplementary Fig. S1). This fusion protein, referred to as H2B-GFP-UVR8 $(2 \mathrm{x})$, did not recruit $\mathrm{mCh}-\mathrm{NLS}-\mathrm{COP} 1^{\mathrm{C} 340}$ to chromatin in the absence of ultraviolet-B. However, after a ultraviolet-B pulse, mCh-NLS$\mathrm{COP} 1^{\mathrm{C} 340}$ localized to chromatin, indicating an interaction with H2B-GFP-UVR8(2x) (Fig. 2b). mCh-NLS-COP1 ${ }^{\mathrm{C} 340}$ remained bound to chromatin for at least $4 \mathrm{~h}$ after exposure to ultraviolet-B (Supplementary Fig. S4a). A mutant H2B-GFP-UVR8 ${ }^{\mathrm{W} 285 \mathrm{~F}}(2 \mathrm{x})$ protein did not interact with $\mathrm{mCh}-\mathrm{NLS}-\mathrm{COP} 1^{\mathrm{C} 340}$ even after exposure to ultraviolet-B (Fig. 2c), while the H2BGFP-UVR $8{ }^{\mathrm{W} 285 \mathrm{~A}}(2 \mathrm{x})$ mutant interacted constitutively with NLS-mCh-COP1 ${ }^{\mathrm{C} 340}$ (Fig. 2d). Similar results were obtained when, in place of a histone $\mathrm{H} 2 \mathrm{~B}$ moiety fused to the $\mathrm{N}$-terminus of UVR8, a histone $\mathrm{H} 3$ moiety was attached to the UVR8 C-terminus (Supplementary Figs S1,S4b).

To quantify the dose required to induce recruitment of NLS-mCh-COP $1{ }^{\mathrm{C} 340}$ to chromatin, we examined over 100 cells, per condition, after administering various doses of ultraviolet-B. We identified three mCh-NLS-COP1 ${ }^{\mathrm{C} 340}$ localization patterns: nucleoplasmic (where mCherry is distributed equally throughout the nucleus); partially nucleoplasmic (where mCherry is principally nucleoplasmic, but partially excluded from the nucleoli) and chromatin-bound (where mCherry colocalized 
with GFP-labelled H2B) (Supplementary Fig. S5). As the dose of ultraviolet- $B$ increased, the percentage of cells exhibiting the chromatin-bound pattern increased, with a plateau being reached at around $25 \mathrm{~J} \mathrm{~m}^{-2}$ (Fig. 2e). In the cells, that were not exposed to ultraviolet-B or that expressed H2B-GFP or H2B-GFP$\mathrm{UVR} 8^{\mathrm{W} 285 \mathrm{~F}}(2 \mathrm{x})$, mCh-NLS-COP1 was nucleoplasmic; whereas, in cells expressing the H2B-GFP-UVR8 ${ }^{\mathrm{W} 285 \mathrm{~A}}(2 \mathrm{x})$ mutant, mCh-NLS-COP1 was constitutively chromatin-bound (Fig. 2e). These results suggest a tight regulation of the UVR8-COP1 interaction by ultraviolet-B light and by amino-acid substitutions affecting UVR8 dimerization in the nuclei of mammalian cells.

Activation of transcription induced by ultraviolet-B light. To establish whether protein-protein interactions relying on ultraviolet-B-dependent UVR8-COP1 heterodimerization could be used to regulate biological activities, we fused the transcription activation domain of NF- $\kappa B$ to UVR8 and the GAL4 DNA-binding domain to $\mathrm{COP} 1^{\mathrm{C} 340}$ (Fig. 3a, Supplementary Fig. S1). We then expressed these proteins together with luciferase reporter plasmids in U2OS cells and measured luciferase activity as a function of exposure to ultraviolet-B light. In the absence of ultraviolet-B, luciferase activity was almost undetectable. In contrast, ultraviolet-B illumination induced a dose-dependent increase in luciferase activity that reached a plateau at a dose of about $25 \mathrm{~J} \mathrm{~m}^{-2}$ (Fig. 3b,c). Similar dose dependency, ultraviolet-B specificity and tightness of regulation were observed in a ultraviolet-B light-switchable gene expression system based on a two-hybrid interaction in yeast (Supplementary Fig. S6).

Temporal and spatial control of protein-protein interactions. In all the experiments described so far, the cells were exposed to ultraviolet-B light using a transilluminator. This means that the cells could not be observed under the microscope at the time of exposure to ultraviolet- $B$ and that, at least, a minute elapsed while transferring the cells from the transilluminator to the microscope. To optimize the method, we incorporated a ultraviolet-B light source on the microscope. The glass used in standard microscope lenses blocks ultraviolet-B. Therefore, a 290-310 nm LED was introduced utilizing a light path that does not involve any of the microscope optics (Fig. 4a). In this setup, the cells were cultured on ultraviolet-B transparent poly-methylmethacrylate (PMMA) coverslips, which were placed in quartz culture dishes.

Using the setup described above, cells expressing H2BGFP-UVR8 $(2 \mathrm{x})$ and $\mathrm{mCh}-\mathrm{NLS}-\mathrm{COP} 1^{\mathrm{C} 340}$ were exposed to ultraviolet- $\mathrm{B}$ for $8 \mathrm{~s}$, while being observed under the microscope. By the end of the $8 \mathrm{~s}$ period, the translocation of mCh-NLSCOP $1^{\mathrm{C} 340}$ from the nucleoplasm to chromatin had already reached a plateau (Fig. 4b, Supplementary Movie 1). To quantify the COP1-UVR8 colocalization, we correlated the intensities of the pixels corresponding to the green (H2B-GFP-UVR8 $(2 \mathrm{x})$ ) and red channels ( $\mathrm{mCh}$-NLS-COP1 ${ }^{\mathrm{C} 340}$ ) over the entire nucleus of a cell before and after exposure to ultraviolet-B. Indeed, ultraviolet$\mathrm{B}$ induced a considerable improvement in the $R^{2}$ linear regression coefficient (Fig. 4c). This improvement was also evident in twodimensional plots of green and red channel intensities of 100 randomly selected pixels before and after ultraviolet-B illumination (Fig. 4c). To determine the minimal ultraviolet-B exposure time needed for the COP1-UVR8 interaction, we repeated the above experiment using subsecond exposure times. After a $0.4 \mathrm{~s}$ ultraviolet-B pulse, there was already partial colocalization of H2B-GFP-UVR8 $(2 \mathrm{x})$ and mCh-NLS-COP1 ${ }^{\mathrm{C} 340}$. The colocalization reached a plateau after an additional $0.4 \mathrm{~s}$ ultraviolet-B pulse (Supplementary Movie 2). a
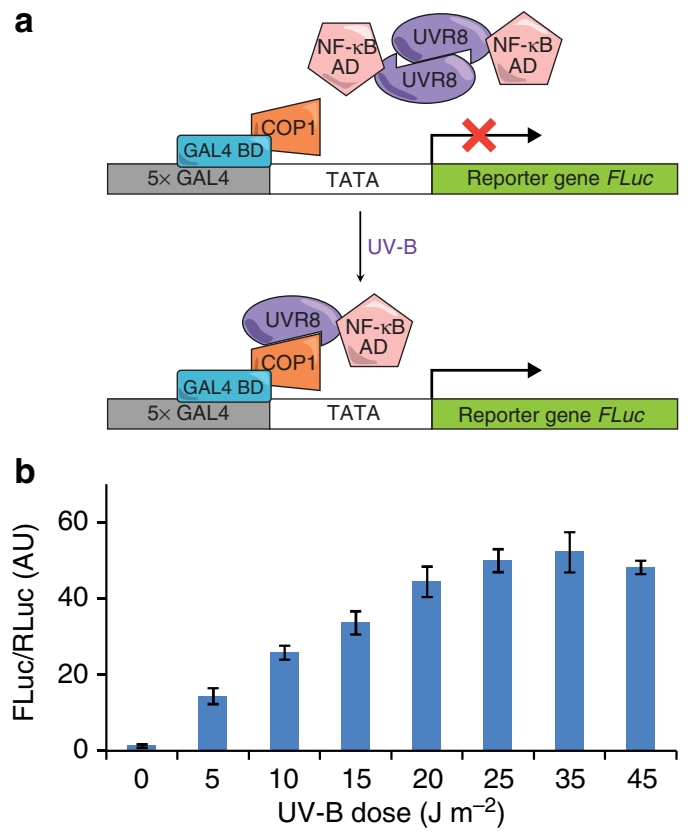

C

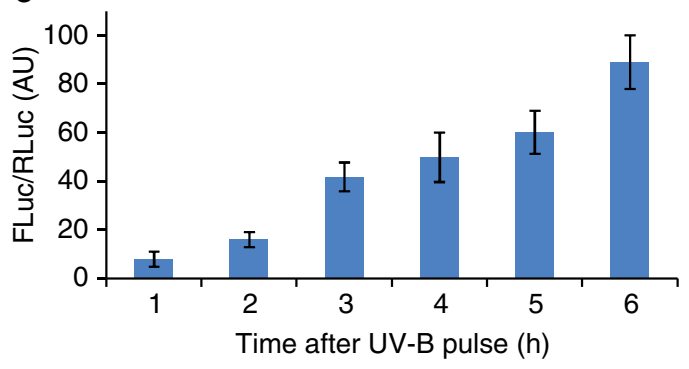

Figure 3 | Ultraviolet-B-dependent induction of transcriptional activity. (a) Schematic representation of the ultraviolet-B-induced interaction of the NF- $\kappa B$ activation domain (AD)-UVR8 fusion protein with the GAL4 DNAbinding domain (BD)-COP1 ${ }^{\mathrm{C} 340}$-fusion protein and subsequent activation of transcription of a firefly luciferase (FLuc) gene driven by five GAL4 response elements. (b,c) Ratio of FLuc to Renilla luciferase (RLuc) activity as a function of exposure to ultraviolet-B. Expression of RLuc is driven by a constitutive promoter. In $\mathbf{b}$, luciferase activity was determined $4 \mathrm{~h}$ after exposure to ultraviolet-B. In c, luciferase activity was determined after exposure to a ultraviolet- $B$ dose of $25 \mathrm{~J} \mathrm{~m}^{-2}$. The bars indicate the means \pm 1 s.e. obtained from four independent experiments.

The ultraviolet-B LED setup described above allowed us to expose subcellular volumes to ultraviolet-B light. This was achieved by placing a collimator in the path of the ultraviolet-B light (Fig. 4a). The collimator that we used contains no optical lenses. It consists of silicon wafers that when stacked against each other form slits, whose width can vary between $1-10 \mu \mathrm{m}$. As the light path through the slits is several $\mathrm{mm}$ long, only rays of light that are parallel to the slits can pass through the collimator. We have previously described the use of this collimator for $\mathrm{X}$-rays (refs 14,15). Here we used a collimator with a light path length of $2.5 \mathrm{~mm}$ and a slit width of $10 \mu \mathrm{m}$ placed about $1 \mathrm{~mm}$ away from the cells. Ultraviolet-B illumination of about half the nucleus of a cell expressing H2B-GFP-UVR8(2x) and mCh-NLS$\mathrm{COP} 1^{\mathrm{C} 340}$ using this setup, resulted in chromatin recruitment of mCh-NLS-COP1 ${ }^{\mathrm{C} 340}$ only in the part of the nucleus that had been exposed to ultraviolet-B light (Fig. 4d, Supplementary Movie 3). Thus, protein-protein interactions can be controlled spatially by controlling the pattern of ultraviolet-B illumination. 
a

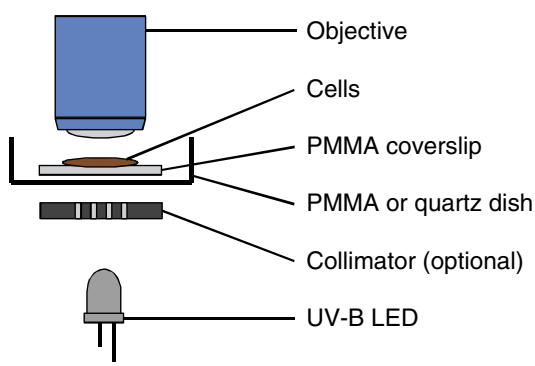

b
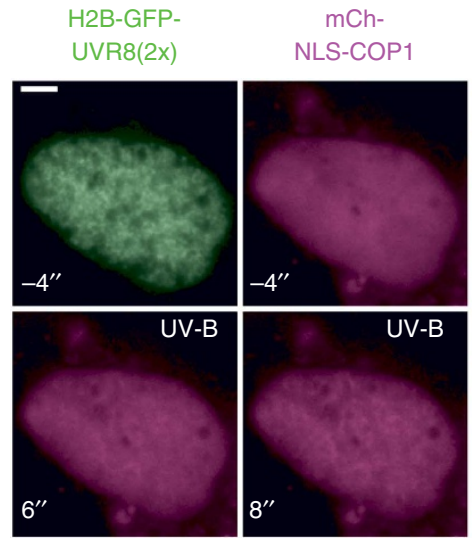

mChNLS-COP1
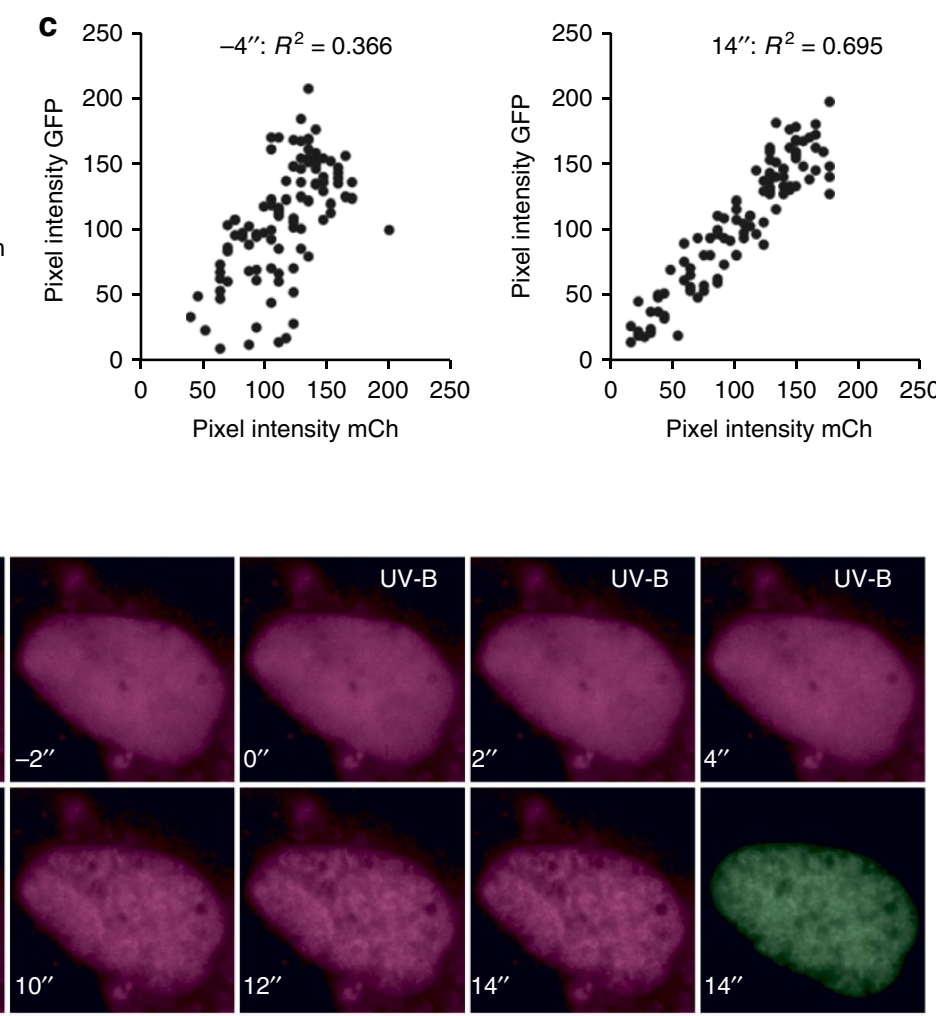

d

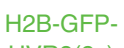
UVR8(2x)

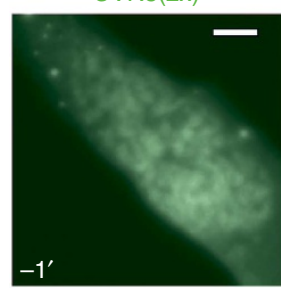

Nis-copt
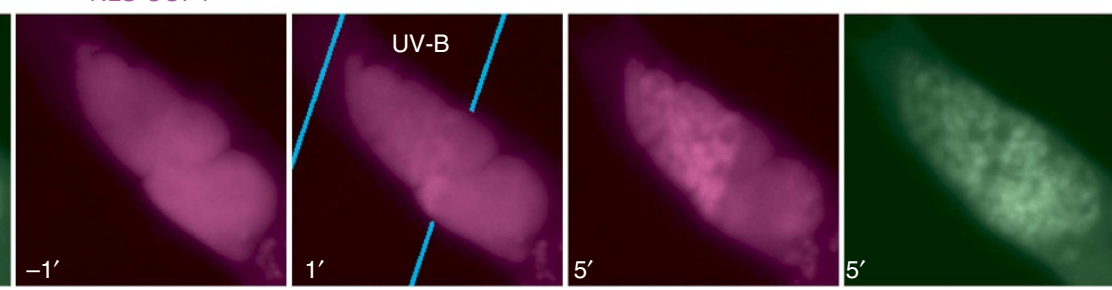

Figure 4 | Induction of H2B-GFP-UVR8(2x) and $\mathbf{m C h}-\mathbf{N L S}-\mathrm{COP}^{\mathbf{C} 340}$ interaction using an LED ultraviolet-B source. (a) Microscope setup. (b) Time-lapse images of U2OS cells expressing H2B-GFP-UVR8(2x) and mCh-NLS-COP1 ${ }^{\mathrm{C} 340}$ using the microscope setup shown in panel (a). The ultraviolet-B LED was turned on for $8 \mathrm{~s}$ (timepoints $0-8^{\prime \prime}$ ). Scale bar, $5 \mu \mathrm{m}$. (c) Linear regression analysis of the pixel intensities for the green $(\mathrm{H} 2 \mathrm{~B}-\mathrm{GFP}-U V R 8(2 \mathrm{x}))$ and red (mCh-NLS-COP1 ${ }^{\mathrm{C} 340}$ ) channels for the images acquired $4 \mathrm{~s}$ before ultraviolet-B illumination (left) and $14 \mathrm{~s}$ (right) after the start of ultraviolet-B illumination. The plots show only 100 randomly selected pixels for clarity. The correlation coefficients $\left(R^{2}\right)$ were calculated using the entire data set. (d) Ultraviolet-B-dependent interaction of H2B-GFP-UVR8(2x) and mCh-NLS-COP1 ${ }^{\mathrm{C} 340}$ in a subnuclear volume. Time-lapse images at the indicated times (in minutes) relative to the ultraviolet-B pulse are shown. The edges of the slit are indicated by the blue lines; the area between these lines was exposed to ultraviolet-B light. Scale bar, $5 \mu \mathrm{m}$.

$\gamma-\mathrm{H} 2 \mathrm{AX}$ foci as indicators of ultraviolet-B-induced DNA damage. One concern of using ultraviolet- $B$ light to induce protein-protein interactions is the potential for ultraviolet-Binduced DNA damage. We therefore examined for the presence of $\gamma-\mathrm{H} 2 \mathrm{AX}$ foci, which are markers of DNA damage ${ }^{16}$, in cells exposed to ultraviolet-B. When exposed to $25 \mathrm{~J} \mathrm{~m}^{-2}$ ultraviolet-B using the transilluminator, the cells showed considerable $\gamma-\mathrm{H} 2 \mathrm{AX}$ signal, as expected (Supplementary Fig. S7). In contrast, when illuminated with the ultraviolet-B LED for $0.5-1 \mathrm{~s}$, an exposure sufficient for the UVR8-COP1 interaction to plateau, the $\gamma-\mathrm{H} 2 \mathrm{AX}$ signal, although detectable, was weak (Supplementary Fig. S7). The wavelength spectrum of ultraviolet-B emitted by the LED was quite narrow $(290-310 \mathrm{~nm})$ and within the range activating UVR8 (ref. 17). In contrast, the ultraviolet-B fluorescent lamps had an emission peak at $312 \mathrm{~nm}$ and a wide emission spectrum ranging from $280-370 \mathrm{~nm}$. UVR8 responds poorly to wavelengths greater than $310 \mathrm{~nm}$, yet these wavelengths can still induce DNA damage ${ }^{17,18}$, thereby explaining why the ultraviolet-B LED performed better than the ultraviolet-B fluorescent lamps present in the transilluminator.

\section{Discussion}

We have developed an optogenetic system to induce proteinprotein interactions using the ultraviolet-B-dependent interaction between UVR8 and COP1. The system works efficiently in mammalian cells and does not require an exogenous chromophore. Further, the activation wavelength is such that the system does not interfere with imaging of the commonly used fluorescent proteins. On the negative side, the interaction between UVR8 and COP1, once induced, is essentially irreversible. Therefore, in its current form the system can be used to induce, but not disrupt, protein-protein interactions. However, in Arabidopsis we have recently demonstrated that the RUP1 and RUP2 proteins 
disrupt the interaction between UVR8 and COP1 (ref. 19), raising the possibility that in the future the optogenetic system described here may be adapted for reversible protein-protein interactions. In certain settings, the irreversible nature of the UVR8-COP1 interaction may be an advantage, as continuous exposure to ultraviolet-B light is not required to maintain the interaction. This is important, as prolonged exposure to ultraviolet- $\mathrm{B}$ light induces DNA damage. Indeed, using LEDs, instead of fluorescent tubes, as the ultraviolet-B source, and short pulses of illumination, we have been able to induce UVR8-COP1 interactions with minimal induction of DNA damage. In principle, the induction of DNA damage could be minimized even further, if the UVR8COP1 interaction were induced by exposing only the cytoplasm to ultraviolet-B. Alternatively, two-photon lasers can excite Trps with 570-600 nm light. Thus, two-photon microscopy may allow high spatial resolution of UVR8 activation, while at the same time limiting ultraviolet-B induced damage. Finally, a unique property of UVR8 among the photoreceptors used so far in optogenetics is its mechanism of activation by monomerization of the UVR8 dimer. This unique mechanism could open up new strategies to control cellular processes by light.

\section{Methods \\ Plasmid construction. The following primers were used (Sigma-Aldrich):}

Primer 1: AAAAAACCCGGGTATAGCAACGGCCTTGCAGATTTTCAATC

Primer 2: AAAAGCGGCCGCTTACGCAGCGAGTACCAGAACTTTGATGG

Primer 3: AAAGGATCCGCGGAGGATATGGCTGCCGACG

Primer 4: AAAAGCGGCCGCTTAAATTCGTACACGCTTGACATCAGTTTG

Primer 5: GTTTTATCTCCCAGATTTCGGGAGGTGCGAGACATACAATGG CATTGACTTCAG

Primer 6: CTGAAGTCAATGCCATTGTATGTCTCGCACCTCCCGAAATCT GGGAGATAAAAC

Primer 7: GTTTTATCTCCCAGATTTCGGGAGGTTTTAGACATACAATGG CATTGACTTCAG

Primer 8: CTGAAGTCAATGCCATTGTATGTCTAAAACCTCCCGAAATCT GGGAGATAAAAC

Primer 9: AAAAGCGGCCGCTTATCCGGAGTCTCCAGAGTCACCAATTCG TACACGCTTGACATCAGTTTGTGG

Primer 10: AAAATCCGGAGATGGTTCTGGTGGTGACGGTTCTGGTGCGG AGGATATGGCTGCCGACG

Primer 11: TTTTTCCGGAGCCTTTGCCACCCTTACCGTCTCCAGAGTCAC CAATTCGTACACGC

Primer 12: AATTCTAAGTGAGTAAAG

Primer 13: GTCCTTTACTCACTTAG

Primer 14: GTACGAATTGGTGACTCTGG

Primer 15: AAAAGCGGCCGCTTATTACTTAAGACCAATTCGTACACGCT TGACATCAGTTTGTGG

AccuPrime Pfx SuperMix (Invitrogen, 12344-040) was used for PCR-amplification. To generate the plasmid encoding mCh-NLS-COP $1^{\mathrm{C} 340}, \mathrm{COP} 1^{\mathrm{C} 340}$ was amplified using primers 1 and 2, and inserted in a pSNV2-CHER-NLS vector with XmaI/NotI. To generate the plasmid encoding GFP-UVR8, UVR8 was amplified using primers 3 and 4, and inserted in a pIRESN2-GFP vector with BamHI/NotI The UVR8 point mutations were introduced with primers 5 and 6 (W285A) and primers 7 and 8 (W285F), using the QuickChange Site-Directed Mutagenesis Kit (Stratagene). To generate the plasmid encoding H2B-GFP-UVR8, UVR8 was amplified using primers 3 and 4 and inserted in a pIRESN2-H2B-GFP vector with BamHI/NotI. The plasmid encoding H2B-GFP-UVR8(2x) was generated in three steps. First a plasmid encoding H2B-GFP-UVR $8^{7 \mathrm{aaLN}}$ was generated, which has a seven amino-acid long flexible linker. For this purpose, a region encoding part of the flexible linker flanked by a BspEI restriction site, a termination codon and a NotI site was introduced by amplifying UVR8 with primers 3 and 9, and insertion into the pIRESN2-H2B-GFP plasmid, using BamHI/NotI. In the second step, a pIRESN2-H2B-GFP-UVR8 ${ }^{16 a a L N}$-UVR8 plasmid was generated by amplifying UVR8 using primers 10 and 4 , and insertion in the pIRESN2-H2B-GFPUVR8 ${ }^{7 a a L N}$ plasmid, using BspEI/NotI. Finally, the plasmid encoding H2B-GFPUVR8 $8^{22 a L N_{-U V R}}$ or, in short, H2B-GFP-UVR8(2x), was generated by amplifying UVR8 $8^{16 a 2 N}$ with primers 3 and 11 and insertion in the pIRESN2-H2B-GFPUVR8 ${ }^{16 a a L N}$-UVR8 plasmid, using BamHI/BspEI. The plasmid encoding GFPUVR8(2x)-H3 was generated in three steps. First, a pIRESN2-GFP-UVR8(2x) plasmid was generated by deleting H2B from pIRESN2-H2B-GFP-UVR8(2x) using annealed primers 12 and 13 and EcoRI/PpuMI. Next, pIRESN2-GFP-UVR8(2x)Afl2 was generated by amplifying pIRESN2-GFP-UVR8(2x) with primers 14 and 15 and inserting into the same vector using BspEI/NotI. Finally, to generate pIRESN2-GFP-UVR8(2x)-H3, a histone H3 insert was cloned into pIRESN2-GFPUVR8(2x)-Afl2, using AflII/NotI. For the mammalian transcription activation assays, UVR8 was amplified using primers 3 and 4 and inserted in the pCMV-AD vector (Stratagene) using BamHI/NotI to generate pCMV-AD-UVR8. COP1 ${ }^{\mathrm{C} 340}$ was amplified using primers 1 and 2, and inserted in pCMV-BD (Stratagene) using Bam HI/NotI to generate plasmid pCMV-BD-COP1 ${ }^{\mathrm{C} 340}$. Plasmids expressing firefly luciferase under the control of GAL4 (pFR-Luc) and Renilla luciferase under the control of a CMV promoter (pRL-CMV) were obtained from Stratagene and Promega, respectively.

Cell culture and transfection. U2OS cells (ATCC) were cultured in Dulbecco's modified Eagle media (DMEM) (Gibco, 11960) supplemented with 10\% FBS (Gibco, 10500) and $1 \times$ penicillin-streptomycin-glutamine (Gibco, 10378). For transfection, cells were grown on $35 \mathrm{~mm}$ culture dishes (200,000 cells per dish) or on custom-made PMMA coverslips in wells of 12 well-plates (100,000 cells per well). The next day, $1 \mu \mathrm{g}$ of each plasmid was added to a $100 \mu \mathrm{l}$ volume of OPTI-MEM I (Gibco, 31985); FuGENE HD (Roche, 04709713001) transfection reagent was subsequently added ( $3 \mu \mathrm{l}$ per $\mu \mathrm{g}$ DNA). After $25 \mathrm{~min}$ incubation at room temperature, this mixture was added to the cells. Two days later, the cells were analysed by fluorescence microscopy before and after exposure to ultraviolet-B.

Ultraviolet-B treatment. To activate UVR8, cells were exposed to ultraviolet-B using either ultraviolet-B fluorescent tubes or a ultraviolet-B LED.

Ultraviolet-B fluorescent tubes. The cells were irradiated by placing the dish with the medium removed, in a CL-1000M Ultraviolet Crosslinker (UVP, 95-023002) equipped with five $8 \mathrm{~W}$ ultraviolet-B lamps (UVP, 34-0042-01) having a peak wavelength of $312 \mathrm{~nm}$ (emission spectrum range: $280-375 \mathrm{~nm}$ ). Unless stated otherwise, the dose administered was $25 \mathrm{Jm}^{-2}$. For the nuclear retention experiment, the cells were analysed by fluorescence microscopy at the indicated times after exposure to ultraviolet-B. For the chromatin recruitment experiment, the cells were analysed by fluorescence microscopy within seconds after exposure to ultraviolet-B.

Ultraviolet- $B$ LED. The cells were grown on custom-made PMMA coverslips placed in quartz culture dishes (Labexchange). A UVTOP295 $295 \mathrm{~nm}$ ultraviolet-B LED (SETI) set to operate at $20 \mathrm{~mA}$ with a typical power output of $0.7 \mathrm{~mW}$ and a peak wavelength of $300 \mathrm{~nm}$ (range $290-310 \mathrm{~nm}$ ) was customized to fit in the condenser carrier of an upright microscope (Zeiss). Optionally, a commercially available collimator $(2.5 \mathrm{~mm}$ light path; $10 \mu \mathrm{m}$ slit width, MicroLeman) was placed between the ultraviolet-B LED and cells.

Imaging. Fluorescence images were acquired using a Zeiss AXIO Imager M.1 upright microscope, equipped with filter sets for DAPI, GFP and RFP (Chroma) and an X-Cite series 120 mercury vapour short arc light source (Lumen Dynamics). Images were captured with an ORCA-ER camera (Hamamatsu). Achroplan $\times 40 / 0.8$ and Achroplan $\times 100 / 1.0$ water immersion objectives were used for live cell imaging and an EC Plan-NEOFLUAR $\times 40 / 1.3$ oil immersion objective was used for imaging of fixed cells. For data processing, the ImageJ (NIH) distribution FIJI was used. Images were enhanced by background subtraction, and linear adjustment of the image brightness and contrast.

Statistical analysis. To determine the nuclear fraction of GFP-UVR8 in cells co-expressing $\mathrm{mCh}$-NLS-COP ${ }^{\mathrm{C} 340}$ before and after exposure to ultraviolet-B light (Fig. 1d), images of 25 cells per condition were acquired. Masks corresponding to the nucleus and plasma membrane of the cell were used to identify pixels corrresponding to the nuclear and total cellular volumes, respectively. The intensity values of these pixels were summed and used to calculate the fraction of nuclear GFP fluorescence for each cell. Means and s.d. of these fractions were then calculated.

To determine the pattern (nucleoplasmic, partially nucleoplasmic or chromatinbound, Fig. 2e) of mCh-NLS-COP1 ${ }^{\mathrm{C} 340}$ localization in U2OS cells expressing mCh-NLS-COP $1{ }^{\mathrm{C} 340}$ together with either H2B-GFP-UVR8(2x), H2B-GFP$\mathrm{UVR}^{\mathrm{W}} 285 \mathrm{~A}(2 \mathrm{x}), \mathrm{H} 2 \mathrm{~B}-\mathrm{GFP}-\mathrm{UVR} 8^{\mathrm{W} 285 \mathrm{~F}}(2 \mathrm{x})$ or H2B-GFP, images of nuclei were acquired before and after exposure to ultraviolet-B. For each condition, five independent experiments were performed and from each experiment images of at least 20 nuclei were acquired. The five experiments were used to calculate means and s.e. of the fraction of nuclei exhibiting a specific mCh-NLS-COP $1^{\mathrm{C} 340}$ localization pattern

To quantify mCh-NLS-COP1 ${ }^{\mathrm{C} 340}$ and H2B-GFP-UVR8(2x) colocalization in $\mathrm{U} 2 \mathrm{OS}$ cells (Fig. 4c), images acquired before $(-4 \mathrm{~s})$ and after (14s) ultraviolet-B illumination were processed using ImageVision software (Silicon Graphics, Inc.). Briefly, a mask corresponding to the nucleus of a cell was used to select only the pixels corresponding to the nuclear area. The pixel intensity values from the red and green channels were used to calculate the $R^{2}$ correlation coefficient using linear regression analysis. The number of pixels used in the analysis was 101,016 for the images acquired before exposure to ultraviolet- $B$ and 103,254 for the images acquired after exposure to ultraviolet-B. For a graphical representation, the intensity values of 100 randomly selected pixels were plotted. 


\section{Two-hybrid transcription activation assays}

Mammalian cells. A plasmid mix consisting of $1 \mu \mathrm{g}$ pCMV-AD-UVR8, $1 \mu \mathrm{g}$ pCMV-BD-COP $1{ }^{\mathrm{C} 340}, 0.2 \mu \mathrm{g}$ pCMV-RL and $0.5 \mu \mathrm{g}$ pFR-Luc was transfected into U2OS cells grown in $3,5 \mathrm{~mm}$ dishes. To measure time dependency, similarlytreated plates of cells were exposed to a ultraviolet-B dose of $25 \mathrm{~J} \mathrm{~m}^{-2}$ using the transilluminator at $48,49,50,51,52$ or $53 \mathrm{~h}$ after transfection. Luciferase activity was determined $1 \mathrm{~h}$ after the last exposure to ultraviolet- $B$. To measure dose dependency, $48 \mathrm{~h}$ after transfection, the cells were exposed to the indicated dose of ultraviolet-B and luciferase activity was determined $4 \mathrm{~h}$ later, using the Dual-Glo Luciferase Assay System (Promega).

Yeast cells. The yeast two-hybrid assay was performed, as previously described ${ }^{7}$.

Ultraviolet-B induced $\gamma-$ H2AX focus formation. U2OS cells grown on PMMA coverslips were irradiated using the ultraviolet-B LED for $0,0.5,1,3,10$ and $30 \mathrm{~s}$, respectively, or exposed to $25 \mathrm{~J} \mathrm{~m}^{-2}$ ultraviolet-B using the ultraviolet-B transilluminator. Two hours after exposure to ultraviolet- $B$, the cells were fixed and processed for immunofluorescence using a rabbit histone H2AX Ser139 phosphospecific antibody (Cell Signaling, 2577, dilution 1:2,500) and secondary Alexa Fluor 594 goat anti-rabbit IgG $(\mathrm{H}+\mathrm{L})$ (Invitrogen, A11012, dilution 1:1,000).

\section{References}

1. Fenno, L., Yizhar, O. \& Deisseroth, K. The development and application of optogenetics. Annu. Rev. Neurosci. 34, 389-412 (2011).

2. Shimizu-Sato, S., Huq, E., Tepperman, J. M. \& Quail, P. H. A light-switchable gene promoter system. Nat. Biotechnol. 20, 1041-1044 (2002).

3. Wu, Y. I. et al. A genetically encoded photoactivatable Rac controls the motility of living cells. Nature 461, 104-108 (2009).

4. Levskaya, A., Weiner, O. D., Lim, W. A. \& Voigt, C. A. Spatiotemporal control of cell signalling using a light-switchable protein interaction. Nature 461, 997-1001 (2009).

5. Yazawa, M., Sadaghiani, A. M., Hsueh, B. \& Dolmetsch, R. E. Induction of protein-protein interactions in live cells using light. Nat. Biotechnol. 27, 941-945 (2009).

6. Kennedy, M. J. et al. Rapid blue-light-mediated induction of protein interactions in living cells. Nat. Methods 7, 973-975 (2010).

7. Rizzini, L. et al. Perception of UV-B by the Arabidopsis UVR8 protein. Science 332, 103-106 (2011).

8. Favory, J. J. et al. Interaction of COP1 and UVR8 regulates UV-B-induced photomorphogenesis and stress acclimation in Arabidopsis. EMBO J 28, 591-601 (2009).

9. Christie, J. M. et al. Plant UVR8 photoreceptor senses UV-B by tryptophanmediated disruption of cross-dimer salt bridges. Science 335, 1492-1496 (2012).

10. Wu, D. et al. Structural basis of ultraviolet-B perception by UVR8. Nature 484, 214-219 (2012).
11. Oravecz, A. et al. Constitutively photomorphogenic1 is required for the UV-B response in Arabidopsis. Plant Cell 18, 1975-1990 (2006).

12. Kaiserli, E. \& Jenkins, G. I. UV-B promotes rapid nuclear translocation of the Arabidopsis UV-B specific signaling component UVR8 and activates its function in the nucleus. Plant Cell 19, 2662-2673 (2007).

13. Yi, C. \& Deng, X. W. COP1 - from plant photomorphogenesis to mammalian tumorigenesis. Trends Cell Biol. 15, 618-625 (2005).

14. Pataky, K. et al. Microcollimator for micrometer-wide stripe irradiation of cells using 20-30 keV X rays. Radiat. Res. 172, 252-259 (2009).

15. Zgheib, O., Pataky, K., Brugger, J. \& Halazonetis, T. D. An oligomerized 53BP1 tudor domain suffices for recognition of DNA double-strand breaks. Mol. Cell. Biol. 29, 1050-1058 (2009).

16. Rogakou, E. P., Pilch, D. R., Orr, A. H., Ivanova, V. S. \& Bonner, W. M. DNA double-stranded breaks induce histone H2AX phosphorylation on serine 139. J. Biol. Chem. 273, 5858-5868 (1998).

17. Brown, B. A., Headland, L. R. \& Jenkins, G. I. UV-B action spectrum for UVR8-mediated HY5 transcript accumulation in Arabidopsis. Photochem. Photobiol. 85, 1147-1155 (2009).

18. Freeman, S. E. et al. Wavelength dependence of pyrimidine dimer formation in DNA of human skin irradiated in situ with ultraviolet light. Proc. Natl Acad. Sci. USA 86, 5605-5609 (1989).

19. Heijde, M. \& Ulm, R. Reversion of the Arabidopsis UV-B photoreceptor UVR8 to the homodimeric ground state. Proc. Natl Acad. Sci. USA 110, 1113-1118 (2013).

\section{Acknowledgements}

The authors thank Marcos González-Gaitán for comments on the manuscript and André Liani for preparing the ultraviolet-B LED and PMMA coverslip setup used in Fig. 4. This work was supported by a grant from the Swiss National Science Foundation to T.D.H.

\section{Author contributions}

R.P.C., R.U. and T.D.H conceived the study; R.P.C. performed all the experiments, except for the yeast two-hybrid analysis, which was performed by R.Y.; R.P.C., R.U. and T.D.H analysed and interpreted the data and wrote the manuscript.

\section{Additional information}

Supplementary Information accompanies this paper at http://www.nature.com/ naturecommunications

Competing financial interests: The authors declare no competing financial interests.

Reprints and permission information is available online at http://npg.nature.com/ reprintsandpermissions/

How to cite this article: Crefcoeur, RP. et al. Ultraviolet-B-mediated induction of protein-protein interactions in mammalian cells. Nat. Commun. 4:1779 doi: 10.1038/ ncomms2800 (2013). 